\title{
MEROMORPHIC MAPPINGS INTO COMPACT COMPLEX MANIFOLDS WITH A GRAUERT POSITIVE BUNDLE OF $q$-FORMS
}

\author{
MYUNG H. KWACK
}

\begin{abstract}
It is shown that a Schwarz lemma, a Picard extension theorem, and a Bloch theorem are valid for meromorphic mappings into compact complex manifolds with (jrauert-positive bundle of $q$-forms.
\end{abstract}

1. Introduction. J. Carlson [1] proved the Ahlfors-Chern-Kobayashi version of Schwarz' lemma for holomorphic mappings from a $q$-dimensional polydisk into a $n$-dimensional compact complex manifold whose bundle of holomorphic $q$-forms is positive in the sense of Grauert. We will show that the Schwarz lemma is also valid for meromorphic mappings and apply the lemma to generalize some classical theorems.

We will prove the following theorems.

THEOREM 1. Let $P^{*}=\left(\Delta^{*}\right)^{k} \times \Delta^{q-k}$ be a punctured polycylinder where $\Delta^{*}=\{z \in$ C: $0<|z|<1\}$ and $\Delta=\{z \in \mathbf{C}:|z|<1\}$ and $W$ be an n-dimensional compact complex manifold such that $\Lambda^{a} T_{W}^{*}$ is positive in the sense of Grauert. Then any nondegenerate meromorphic mapping $F: P^{*} \rightarrow W$ extends to a meromorphic mapping from $\Delta^{q}$ into $W$.

Theorem 1 was proved by $\mathbf{J}$. Carlson [1] when $f$ is holomorphic.

THEOREM 2. Let $W$ be as in Theorem 1 and $\bar{y}$ be a family of meromorphic mappings $f: \Delta^{q} \rightarrow W$ such that $f$ is holomorphic in a neighborhood of zero and $|J f(0)| \geqslant 1$. Then given an arbitrary sequence of mappings $\left\{f_{k}^{\#}\right\} \subset \widetilde{F}$, there is a subsequence $\left\{f_{k}\right\}$ of $\left\{f_{k}^{\#}\right\}$ which converges to a meromorphic mapping $g \in \bar{y}$. In addition there is a number $\rho$ with $0<\rho \leqslant 1$ such that the restrictions of the mappings $\left\{f_{k}\right\}$ to $\Delta^{q}(\rho)=\left\{z \in \Delta^{q}\right.$ : $\left.\left|z_{i}\right|<\rho, i=1, \ldots, q\right\}$ are holomorphic and such that the sequence $\left\{f_{k}\right\}$ converges to $g$ uniformly on compact subsets of $\Delta^{q}(\rho)$.

$|J f(0)|$ is defined by measuring $f_{*}\left(\partial / \partial z_{1} \wedge \cdots \wedge \partial / \partial z_{q}\right)$ with respect to a metric $\nu$ in $\Lambda^{q} T_{W}^{*}$.

To state the next theorem we define a univalent ball for a mapping.

Let $f: \Delta^{q}(\rho) \rightarrow N$ be a meromorphic mapping into a complex manifold $N$ with a Hermitian metric $d s_{N}^{2}$. Suppose that there exists a submanifold $M$ of $N$ such that

Received by the editors April 28, 1982 and, in revised form, July 12, 1982.

1980 Mathematics Subject Classification. Primary 32A10, 32A30; Secondary 32A10.

(5) 1983 American Mathematical Society 0002-9939/82/0000-0737/\$02.00 
$f(0) \in M$ and such that $f$ maps some open set $U$ about 0 univalently (biholomorphically) onto an open ball $B(f(0), r)=\left\{q \in M: d_{M}(f(0), q)<r\right\}$ where $d_{M}$ is the distance on $M$ induced from the Hermitian metric $d s_{N}^{2}$. In this case the open ball $B(f(0), r)$ will be called a univalent ball for $f$ about $f(0)$ of radius $r$.

THEOREM 3. Let $W$ and $\bar{y}$ be as in Theorem 2. Then there exists an absolute constant $r>0$ such that there is a univalent ball of radius $r$ about $f(0)$ for every $f \in \bar{y}^{\prime}$.

Theorems 2 and 3 were proved by P. Griffiths [2] when $W$ is a canonical algebraic manifold and $\bar{y}$ is a family of holomorphic mappings.

THEOREM 4. Let $W$ be an n-dimensional compact complex manifold such that $\Lambda^{\prime \prime} T_{W}^{*}$ is positive in the sense of Grauert and $x_{0} \in W$. Let $f: W \rightarrow W$ he a meromorphic mapping such that $f$ is holomorphic in a neighborhood of $x_{0}$ and $f\left(x_{0}\right)=x_{0}$.

Then $f$ satisfies the following:

(1) $\left|J f\left(x_{0}\right)\right| \leqslant 1$;

(2) $f_{*}: T_{x_{0}}(W) \rightarrow T_{x_{1}}(W)$ is the identity transformation if and only if $f$ is the identity transformation of $W$;

(3) $\left|J f\left(x_{0}\right)\right|=1$ if and only if $f$ is a biholomorphic mapping.

Theorem 4 was proved independently by W. Kaup [3] and $\mathrm{H}$. Wu [7] when $W$ is a complete hyperbolic manifold and $f$ is holomorphic.

2. Proof of theorems. Let $W$ be a compact complex manifold such that $\Lambda^{4} T_{W}^{*}$ is positive in the sense of Grauert. We choose $/$ large enough so that $S^{l}\left(\Lambda^{4} T_{W}^{*}\right)$ is very ample where $S^{l}(E)$ denotes the $l$ th symmetric power of the bundle $E$. Let $\Gamma$ be the vector space of holomorphic sections of $S^{l}\left(\Lambda^{4} T_{w}^{*}\right)$. Evaluation of a section on the fiber gives the exact sequence

$$
0 \rightarrow F \rightarrow W \times \Gamma \rightarrow S^{\prime}\left(\lambda^{\prime} T_{W}^{*}\right) \rightarrow 0
$$

which defines an imbedding of $W$ into a Grassmannian $\operatorname{Gr}(m, N)$ by sending $x$ to the subspace $F_{x}$ of $\Gamma$. We choose a basis $w_{1}, \ldots, w_{N}$ for $\Gamma$ and fix an imbedding $\gamma$ : $W \rightarrow \operatorname{Gr}(m, N)$ determined by the basis. We also fix a metric in $S^{\prime}\left(\Lambda^{q} T_{W}^{*}\right)$ by $\nu=w_{1} \wedge \bar{w}_{1}+\cdots+w_{N} \wedge \bar{w}_{N}$.

Let $\Delta^{q}(R)=\left\{z \in \mathbf{C}^{q}:\left|z_{i}\right|<R, i=1, \ldots, q\right\}$. The Poincaré volume element

$$
\mu_{R}=(\sqrt{-1})^{q} a^{q} R^{2 q} \frac{d z_{1} \wedge d \bar{z}_{1} \wedge \cdots \wedge d z_{q} \wedge d \bar{z}_{q}}{\prod_{j=1}^{q}\left(R^{2}-\left|z_{j}\right|^{2}\right)^{2}}
$$

is invariant under all complex automorphisms of $\Delta^{q}(R)$. Let $f: \Delta^{q}(R) \rightarrow W$ be a meromorphic mapping. Then $f$ is holomorphic on $\Delta^{q}(R)-A$ where $A$ is an analytic set with codimension $A \geqslant 2$. Thus $f^{*} w_{j}$ is holomorphic on $\Delta^{q}(R)-A$ for $j=1, \ldots, N$. Hartogs' theorem implies that $f^{*} w_{j}$ is holomorphic on $\Delta^{q}(R)$. This implies that $\left(f^{*} \nu\right)^{1 / l}$ is a pseudo-metric on $\Lambda^{q} T_{\Delta^{q}(R)}^{*}$ and is a metric on $\Delta^{q} T_{B}^{*}$ where $B=\{p \in$ $\left.\Delta^{q}(R):\left(f^{*} \nu\right)^{1 / l}(p) \neq 0\right\}$. Also $f$ is locally a holomorphic diffeomorphism of $B$ onto $f(B)$. Now the proof of J. Carlson [1, Proposition 2] for holomorphic mappings can be applied to $\left(f^{*} \nu\right)^{1 / l}$ to give 
Proposition 1. Let $f: \Delta^{q}(R) \rightarrow W$ be a meromorphic mapping. Then a suitable normalization constant in the Poincaré volume element $\mu_{R}$ can be chosen independently of $f$ such that

$$
\left(f^{*} \nu\right)^{1 / I} \leqslant \mu_{R}
$$

Proof of Theorem 1. Let $\lambda: \Delta^{q} \rightarrow P^{*}$ be the universal covering map. Since the Poincare volume $\mu$ on $\Delta^{q}$ is invariant under covering transformations, it descends to give a volume form

$$
\mu_{P^{*}}=(\sqrt{-1})^{q} a^{q} \frac{d z_{1} \wedge d \bar{z}_{1} \wedge \cdots \wedge d z_{1} \wedge d \bar{z}_{q}}{\prod_{j=1}^{q}\left(\left|z_{j}\right| \log \left|z_{j}\right|^{2}\right)^{2}}
$$

on $P^{*}$. Since $\left((f \circ \lambda)^{*} \nu\right)^{1 / l} \leqslant \mu$ on $\Delta^{q}$, we get $\left(f^{*} \nu\right)^{1 / l} \leqslant \mu_{P^{*}}$.

For the basis elements $w_{1}, \ldots, w_{N}$ of $\Gamma$, the space of holomorphic sections of $S^{l}\left(\Lambda^{q} T_{W}^{*}\right)$, write $f^{*} w_{i}=\tilde{f}_{i}\left(d z_{1} \wedge \cdots \wedge d z_{q}\right)^{l}$ where $\tilde{f}_{i}$ are holomorphic functions on $P^{*}$.

Then $f^{*} \nu=\Sigma f^{*}\left(w_{1} \wedge \bar{w}_{i}\right)=\Sigma\left|\tilde{f}_{i}\right|^{2}\left(d z_{1} \wedge \cdots \wedge d \bar{z}_{q}\right)^{\prime}$. Since

$$
\frac{1}{N}\left|\tilde{f}_{i}\right|^{2 / l} \leqslant \frac{1}{N} \sum\left|\tilde{f}_{i}\right|^{2 / l} \leqslant\left(\frac{1}{N} \sum\left|\tilde{f}_{i}\right|^{2}\right)^{1 / l}
$$

we have

$$
\frac{1}{N}\left(f^{*}\left(w_{i} \wedge \bar{w}_{\imath}\right)\right)^{1 / 1} \leqslant\left(\frac{1}{N}\right)^{1 / 1}\left(f^{*} \nu\right)^{1 / 1} \leqslant\left(\frac{1}{N}\right)^{1 / 1} \mu_{P *}
$$

It follows that

$$
\left|\tilde{f}_{1}\right|^{2 / l} \leqslant N\left(\frac{1}{N}\right)^{1 / l} a^{q} \prod_{j=1}^{q} \frac{1}{\left(\left|z_{j}\right| \log \left|z_{j}\right|^{2}\right)^{2}}
$$

and

$$
\left|\tilde{f}_{i}\right|^{2} \leqslant c \prod_{j=-1}^{4} \frac{1}{\left(|z,| \log \left|z_{1}\right|^{2}\right)^{2 l}}
$$

where $c$ is a constant. Thus $\left(z_{1} \cdots z_{q}\right)^{\prime} \tilde{f}_{i}(z)$ is holomorphic in $P^{*}$ and locally bounded on $P^{*}$ and therefore holomorphic on $\Delta^{q}$. Thus $f^{*} w$ is meromorphic on $\Delta^{4}$ for $i=1, \ldots, N$ and we obtain our theorem.

Proof of Theorem 2. Let $w$ be one of the basis elements $w_{1}, \ldots, w_{N}$ of $\Gamma$ and write $\left(f_{k}^{\#}\right)^{*} w=\tilde{f}_{k}^{\#}\left(d z_{1} \wedge \cdots \wedge d z_{q}\right)^{l}$ where $\left\{\tilde{f}_{k}^{\#}\right\}$ are holomorphic functions on $\Delta^{q}$. Then as in Theorem 1, we get

$$
\frac{1}{N}\left(\left(f_{k}^{\#}\right)^{*}(w \wedge \bar{w})\right)^{1 / l} \leqslant\left(\frac{1}{N}\right)^{1 / l}\left(f^{*} \nu\right)^{1 / l} \leqslant\left(\frac{1}{N}\right)^{1 / l} \mu
$$

where the last inequality follows from Proposition 1. Thus

$$
\left|\tilde{f}_{k}^{\#}\right|^{2} \leqslant c \prod_{j=1}^{q}\left(\frac{1}{1-\left|z_{j}\right|^{2}}\right)^{2 \prime}
$$


where $c$ is a constant. It follows that $\tilde{f}_{k}^{*}$ are locally uniformly bounded. Thus the sequence $\left\{f_{k}^{\#}\right\}$ has a subsequence $\left\{\tilde{f}_{k}\right\}$ which converges uniformly on compact sets of $\Delta^{q}$ to a holomorphic function $\tilde{h}$. Then the corresponding holomorphic forms $\left\{f_{k}^{*} w\right\}$ converge uniformly on compact sets to $h(z)\left(d z_{1} \wedge \cdots \wedge d z_{q}\right)^{\prime}$.

By taking a subsequence if necessary we may assume that a subsequence $\left\{f_{k}\right\}$ of $\left\{f_{k}^{\#}\right\}$ has been selected so that the pull backs $f_{k}^{*} w_{\alpha}$ converge uniformly on compact sets to a holomorphic form $\phi_{\alpha}$ for $\alpha=1, \ldots, N$. Then the forms $\phi_{\alpha}, \alpha=1, \ldots, N$, define a meromorphic mapping $f: \Delta^{q} \rightarrow W$ by considering the imbedding of $W$ in $\operatorname{Gr}(m, N)$ determined by $w_{1}, \ldots, w_{N}$. Since $|J f(0)| \geqslant 1, f$ will be holomorphic in $\Delta^{q}(\rho)$ for some $0<\rho \leqslant 1$. There exists a number $\rho_{0}, 0<\rho_{0} \leqslant \rho$ such that $\left\{f_{k}\right\}$ is holomorphic in $\Delta^{q}\left(\rho_{0}\right)$ for $k=1,2, \ldots$. For, otherwise there is a sequence of points $\left\{p_{k}\right\}$ converging to zero such that $f_{k}$ is meromorphic at $p_{k}$. Then $\lim _{k \rightarrow \infty} \tilde{f}_{k, \alpha}\left(p_{k}\right)=$ $\tilde{\phi}_{\alpha}(0)=0$ for some $\alpha$ 's where $\tilde{f}_{k, \alpha}$ and $\tilde{\phi}_{\alpha}$ are defined by

$$
f_{k}^{*} w_{\alpha}=\tilde{f}_{k, \alpha}\left(d z_{1} \wedge \cdots \wedge d z_{q}\right)^{\prime}
$$

and

$$
\phi_{\alpha}=\tilde{\phi}_{\alpha}\left(d z_{1} \wedge \cdots \wedge d z_{q}\right)^{\prime} .
$$

It follows that $f$ is meromorphic at zero, a contradiction.

Proof of Theorem 3. Assume that the theorem is false. Then there exists a sequence $\left\{f_{k}^{\#}\right\} \subset \mathcal{F}$ such that the radius of a maximal univalent ball for $f_{k}^{\#}$ about $f_{k}^{\#}(0)$ approaches zero as $k \rightarrow \infty$. By Theorem 2 , there is a subsequence $\left\{f_{k}\right\}$ of $\left\{f_{k}^{\#}\right\}$ which converges to an $f \in \mathscr{F}$ and there is $\rho, 0<\rho \leqslant 1$, such that the mappings $\left\{f_{k}\right\}$ are holomorphic in $\Delta^{q}(\rho)$ and converge uniformly on a compact subset of $\Delta^{q}(\rho)$.

There exists $\rho_{0}, 0<\rho_{0}<\rho$, with the following properties:

(1) there is a univalent ball $B$ of radius $r>0$ for $f: \Delta^{q}\left(\rho_{0}\right) \rightarrow W$ about $f(0)$, and

(2) $m=\inf _{p \in \partial B} d_{W}(f(0), p)>0$, where $d_{W}$ is the distance on $W$ induced from a Hermitian metric on $W$.

Let $0<4 \varepsilon<m$ and let

$$
\begin{aligned}
& P^{\prime}=\left\{q \in W: d_{W}(f(0), q)<m-2 \varepsilon\right\}, \\
& P^{\prime \prime}=\left\{q \in W: d_{W}(f(0), q)<m-4 \varepsilon\right\} .
\end{aligned}
$$

Also let $A$ (respectively $A^{\prime}$ ) be the open set in $\Delta^{q}\left(\rho_{0}\right)$ which contains 0 and which are mapped by $f$ univalently onto $B$ (respectively $P^{\prime} \cap B$ ).

Let $B_{j}$ be the maximal univalent ball about $f_{j}(0)$, say of radius $r_{j}$, for $f_{j}$ : $\Delta^{q}\left(\rho_{0}\right) \rightarrow W$. Since $d_{j}=\sup _{p \in B_{j}} d_{W}(f(0), p) \leqslant r_{j}, \lim _{j \rightarrow \infty} d_{j} \leqslant \lim _{j \rightarrow \infty} r_{j}=0$. Let $A_{j}$ be the open neighborhood of 0 in $\Delta^{q}\left(\rho_{0}\right)$ which are mapped univalently onto $B_{j}$ by $f_{j}$. Then $\partial A_{j}$ must contain a boundary point of $\Delta^{q}\left(\rho_{0}\right)$ or a point $p_{j}$ at which $J f_{j}\left(p_{j}\right)=0$. Since $\lim _{j \rightarrow \infty} d_{j}=0$ and $\lim _{j \rightarrow \infty} f_{j}(0)=f(0)$, we can choose an integer $N_{1}$ : so large that $B_{j} \subset P^{\prime \prime}$ for $j \geqslant N_{1}$. Also, since $\overline{A^{\prime}}$ is compact, there is an integer $N_{2}$ such that

$$
\sup _{x \in \bar{A}^{\prime}} d_{W}\left(f(x), f_{j}(x)\right) \leqslant \varepsilon \quad \text { if } j \geqslant N_{2} .
$$


We claim that $A_{j} \subset A^{\prime}$ if $j \geqslant \max \left\{N_{1}, N_{2}\right\}$. For if not there is a point $x \in A_{j} \cap \partial A^{\prime}$. Then $f_{j}(x) \in B_{j} \subset P^{\prime \prime}$ and $d_{w}\left(f_{j}(x), \partial\left(P^{\prime} \cap B\right)\right) \geqslant \varepsilon$. On the other hand $f(x) \in f\left(\partial A^{\prime}\right)=\partial\left(P^{\prime} \cap B\right)$ and so $d_{w}\left(f_{j}(x), \partial\left(P^{\prime} \cap B\right)\right) \leqslant d_{w}\left(f_{j}(x), f(x)\right)+$ $d_{w}\left(f(x), \partial\left(P^{\prime} \cap B\right)\right) \leqslant \varepsilon$. This is a contradiction. Therefore $A_{j} \subset A^{\prime}$. Since $\bar{A}, \subset \overline{A^{\prime}}$ for $j \geqslant \max \left\{N_{1}, N_{2}\right\}, \bar{A}_{j}$ contains no boundary point of $\Delta^{q}\left(\rho_{0}\right)$. Therefore there is a point $p_{j} \in \partial A_{j}$ such that $J f_{j}\left(p_{j}\right)=0$ for $j \geqslant \max \left\{N_{1}, N_{2}\right\}$. The set $\left\{p_{j}\right\}$ has a subsequence $\left\{p_{j_{k}}\right\}$ which converge to a point $p \in \overline{A^{\prime}}$.

By the uniform convergence of $\left\{f_{j}\right\}$ to $f$ on $\overline{A^{\prime}}$, we have $\lim _{k \rightarrow \infty} J f_{j_{k}}\left(p_{j_{h}}\right)=J f(p)$ $=0$. This is impossible since $p \in \overline{A^{\prime}} \subset A$ and $f$ is univalent on $A$. This finishes the proof.

In view of Theorem 2 and Theorem 3, Theorem 4 can be proved as in Theorem B in $\mathrm{H}$. Wu [7] or in Kwack [6].

\section{REFERENCES}

I. J. Carlson, Some degeneracy theorems for entire functions with values in an algehraic tarietr. Trans. Amer. Math. Soc. 168 (1972), 273-301.

2. P. A. Griffiths, Holomorphic mappings into canonical algebraic varieties, Ann. of Math. (2) 93 (1971). 439-458.

3. W. Kaup. Reelle Transformationsgruppen und invariante Metriken auf komplexen Räumen. Invent. Math. 3 (1967), 43-70

4. S. Kobayashi, Hyperholic manifolds and holomorphic mappings. Monograph. Dekker. New York. 1970.

5. S. Kobayashi and T. Ochiai, Mappings into compact complex manifolds with negative first Chern class. J. Math. Soc. Japan 23 (1971), 137-148.

6. M. Kwack. A Schwarz lemma for canonical algebraic manifold. Proc. Amer. Math. Soc. 41 (1973), 219-222.

7. H. Wu, Normal families of holomorphic mappings, Acta Math. 119 (1967), 193-233.

Department of Mathematics, Howard University, Washington, D.C. 20059 